\title{
Une catégorisation des maquettes BIM au sein des modèles scientifiques
}

\section{A categorization of BIM models within scientific models}

\author{
Aurélien Catros* \\ Doctorant, dir. J-P Chupin et B. Helal, Université de Montréal, Faculté de l'aménagement, École \\ d'Architecture, 2940 Chemin de la Côte-Sainte-Catherine, Montréal, QC H3T 1B9, Canada
}

\begin{abstract}
Résumé. À la faveur d'un regard rétrospectif sur l'histoire des modèles d'architecture et des modèles scientifiques depuis le Quattrocento, le présent article se propose de situer les modèles BIM utilisés en architecture au sein des catégorisations générales des modèles proposées par $\mathrm{M}$. Weisberg et $\mathrm{M}$. Echenique. Par là même, il contribue à préciser leur définition en les situant dans un cadre épistémologique plus large.
\end{abstract}

Mots-clés. Building Information Modeling, Maquette, Représentation, Simulation, Classification

\begin{abstract}
Through a look at the history of architectural and scientific models since the Quattrocento, this article aims to situate the BIM models used in architecture within the general categorizations of models proposed by $\mathrm{M}$. Weisberg and M. Echenique. Its main contribution is to help to clarify their definition by placing them in a broader epistemological framework.
\end{abstract}

Keywords. Building Information Modeling, Models, Representation, Simulation, Classification

\section{Introduction}

La technologie BIM, acronyme de «building information modeling » s'est progressivement imposée aux acteurs de l'industrie de la construction au cours des deux dernières décennies. Pour distinguer des pratiques qui n'ont parfois que le terme en commun, on échelonne les différentes pratiques de modélisation en 4 catégories (Chudy et Gasparek 2017). Le «BIM $0 »$ correspond aux pratiques dites de dessin assisté par ordinateur (DAO) - CAD en anglais - qui se sont démocratisées dans les années 1990 et n'est pas véritablement considéré comme de la modélisation intégrée. À l'autre extrémité du spectre, le «BIM 3 » est une collaboration complète entre tous les acteurs du projet au

\footnotetext{
*Corresponding author: aurelien.catros@umontreal.ca
} 
moyen d'un modèle numérique partagé unique conservé dans une base de données et modifiable par tous. Objectif de la plupart des professionnels, qui, dans les faits, sont encore à «BIM 1 » ou «BIM 2 », des niveaux intermédiaires du processus dans lesquels les modèles $3 \mathrm{D}$ ne sont pas ou pas entièrement partagés entre les différents membres de l'équipe du projet.

Confondre la pratique qu'est le «building information modeling» avec le «building information model $\gg$ qui est plutôt l'outil autour duquel s'organise cette pratique, suscite son lot de problème. Il sera ici question des maquettes numériques utilisées dans un processus de BIM 3 qui constitue selon toute probabilité l'avenir du secteur (Eastman et al. 2018). Nous les définissons comme des représentations numériques du bâtiment à travers ses composants par des objets numériques 3D dotés d'attributs graphiques géométriques et de données quantitatives qui décrivent leur fonctionnement et peuvent être analysées. La modification des données de l'un des composants est possible par différents acteurs du processus et est automatiquement répercutée dans toutes ses représentations. Ces dernières restent, par conséquent, toujours cohérentes entre elles.

Considérées comme l'aboutissement d'un processus de transition vers le numérique entamé à la fin du siècle dernier, ces maquettes constituent des objets théoriques à part entière dont la nature épistémologique reste imprécise. Ces objets commencent pourtant à être utilisés à des fins scientifiques, notamment dans le domaine de la reconstitution historique (Pocobelli et al. 2018). Dès lors, il convient d'interroger leur caractère scientifique à l'aulne de l'histoire des modélisations architecturales. Ce regard rétrospectif permettra de contribuer à une catégorisation de ces représentations au sein de ce qui pourrait être une «théorie générale des modèles ». Il ne s'agit pas ici d'une catégorisation définitive mais bien d'une proposition visant à montrer que les avancées technologiques en architecture, en général, et des maquettes BIM, en particulier - aussi prometteuses qu'elles puissent être - ne devraient pas se faire sans efforts de théorisation comparable à l'envergure qu'elles prennent au sein des pratiques contemporaines.

\section{Classification des modèles : de la représentation à la simulation}

Dans les faits, les maquettes BIM permettent des usages assez proches des maquettes analogiques qui les ont précédées et qu'elles remplacent peu à peu sans toutefois parvenir à les supplanter totalement. Elles disposent pourtant de plusieurs caractéristiques singulières dont certaines renvoient à des modèles aussi éloignés de l'architecture que les simulations météorologiques, par exemple, avec le découpage d'un objet global en sous-éléments discrets. Les maquettes BIM intègrent aussi des concepts géométriques et leur représentation spatiale, ce qui tend à les rapprocher des modèles mathématiques logiques. Il est donc difficile de comprendre la nature de ces objets sans la clarification d'un cadre théorique épistémologique. 


\subsection{Définitions \& classifications générales}

Nous considérerons ici les modèles comme des "opérations contrôlées de négligence $\dagger^{\dagger} "$, des constructions qui hiérarchisent les données d'un problème, soulignant les caractéristiques principales et négligeant de fait tous les aspects considérés secondaires à sa résolution (Nouvel 2002). Ce sont des «représentation de la réalité, dans laquelle la représentation est l'expression de certaines caractéristiques pertinentes de la réalité observée et où la réalité est constituée d'objets ou de systèmes qui existent, ont existé ou pourraient exister. "** (Echenique 1968).

Ces définitions ont l'avantage d'ouvrir à la fois sur les modèles dits scientifiques, et sur les maquettes BIM utilisés en architecture. Seule la nature des problèmes auxquels ils proposent une résolution diffère. Les premiers s'attachent à comprendre un phénomène et le problème est, réciproquement, l'incompréhension - voire même la mécompréhension dudit phénomène. Les seconds participent à la construction d'un bâtiment qui est considérée en soi comme la résolution d'un problème, de sa conception à son édification.

Dans les années 1960, deux épistémologues, Max Black et Mary Hesse, ont proposé des pistes qui permettent de tracer les contours d'une classification générale des modèles architecturaux comme scientifiques - dans laquelle nous pourrions placer les maquettes numériques d'architecture les plus récentes.

Max Black considère les modèles en fonction du rapport qu'ils entretiennent avec leur réalité observée, c'est-à-dire la modification réalisée pour passer de l'objet ou du système à son modèle (Black 1962). Il distingue ainsi quatre modèles archétypiques. Le premier est celui des modèles produits par changement d'échelle qui, bien que toujours imparfaits, rendent accessibles les phénomènes trop grands ou trop petits pour être observés et compris dans la nature. Le second archétype est celui des modèles produits par changement de support qui fournissent des «hypothèses plausibles et non des preuves » et qu'il nomme modèles analogues. Le troisième archétype est celui des modèles mathématiques qui opèrent par simplification des phénomènes et traitement mathématique sans jamais fournir d'explication causale (et, à ce titre, ne doivent pas être confondus avec les modèles analogues). Enfin, le quatrième archétype est celui des modèles théoriques, spéculations qui modifient la représentation même du phénomène : ce sont des fictions heuristiques qui inventent l'objet qu'elles modélisent pour atteindre une explication. Ces derniers modèles ne doivent pas être confondus avec les modèles analogues imaginaires des architectes, qui, bien que spéculatifs, n'admettent aucune dimension explicative.

À l'exception des modèles obtenus par changement d'échelle, ces archétypes correspondent peu ou prou aux trois grands types de modèles - ou théories - définis et popularisés par Mary Hesse (Hesse 1970). Ils sont hiérarchisés selon leur pouvoir prédictif, d'une part, et selon la justification des critères choisis, d'autre part. Les modèles analogues sont décrits comme des idéalités en ce qu'ils dérivent de théories causales déjà établies. Les modèles conceptuels - modèles théoriques chez Black -, moins robustes parce que formulés ex nihilo, ne seront mobilisés que lorsqu'aucun modèle analogue n'est envisageable. Enfin, si aucun modèle conceptuel ne peut être établi, on s'en tiendra à une théorie formelle, autrement dit, une hypothèse strictement mathématique dont on ne saura donner aucune explication conceptuelle et dont le seul avantage est sa correspondance stricte aux données récoltées - raison même de sa constitution.

\footnotetext{
$\dagger$ Nouvel, P., p 193.

* "A model is a representation of a reality, where representation is the expression of certain relevant characteristics of the observed reality and where reality consist of the objects or systems that exist, have existed, or may exist.”, Echenique, M., Op. Cit., p. 1. Notre traduction.
} 
On comprendra que ces catégories ne semblent pas suffisantes pour distinguer les maquettes analogiques de leurs équivalents numériques - BIM ou autre - qui ne se limitent pas à une différence de rapport à la réalité qui pourrait être celle du projet, ni à la puissance de leurs pouvoirs prédictifs - ou prescriptifs - respectifs.

\subsection{Catégorisation des modèles en architecture}

Inspirée des catégorisations de Black et Hesse, les architectes ont proposé dès la fin des années 1960 une classification tridimensionnelle générale des modèles (Echenique 1968) qui sera véritablement utilisée pour étudier les modélisations architecturales près de quatre décennies plus tard (Dunn 2007). Chaque modèle s'y trouve décrit par trois caractéristiques précises : l'objectif ("pour quoi le modèle a-t-il été fait? »), le support (" de quoi le modèle est-il fait ? ») et le rapport au temps ("comment le modèle prend-il en compte le facteur temps?») . $^{\S}$

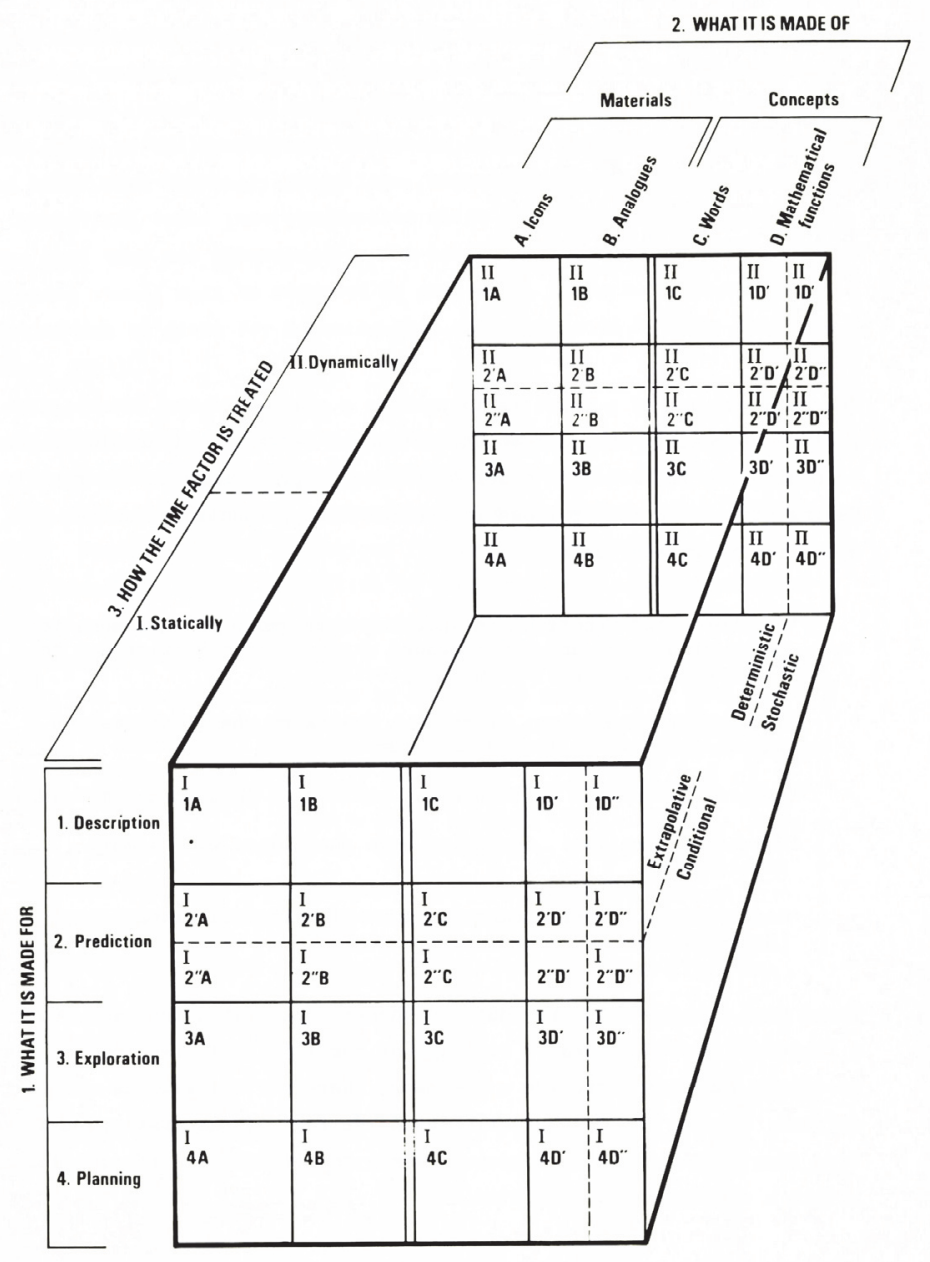

Figure 1. Classification tridimensionnelle générale des modèles, M. Echenique, 1968

§ "What is it made for?, what is it made of?, How the time factor is treated?" Echenique, M., notre traduction. 
Echenique identifie quatre objectifs des modèles: la description, la prédiction, l'exploration - c'est-à-dire la spéculation - et l'optimisation. Cette première caractéristique permet la distinction des différentes itérations que connaît un modèle BIM tout au long du processus de conception. La seconde caractéristique amenée par cette classification est le support du modèle, c'est-à-dire, le « matériau » qui le constitue et par lequel il est compris. Un modèle peut reposer sur des icônes, un matériau réel similaire au phénomène observé, des analogues, un matériau différent qui représente celui du phénomène observé, des mots ou des fonctions mathématiques. L'utilisation quasi systématique de l'informatique pour la construction de tous types de modèles - y compris les modèles matériels avec l'impression $3 \mathrm{D}$ - dans les dernières décennies a montré l'importance de cette caractéristique. Michael Weisberg en propose une déclinaison plus récente dans laquelle il sépare simplement modèles réels et concrets, modèles mathématiques et modèles informatiques quand bien même ils poursuivraient des objectifs similaires (Weisberg 2013). La troisième caractéristique est le rapport qu'entretient le modèle avec la temporalité des phénomènes qu'il observe. Peu développée par Echenique, elle permet de séparer les modèles dynamiques, qui évoluent au cours du temps, des modèles statiques.

Cette décomposition, bien qu'assez systématique, a le mérite de clarifier les limites des catégories proposées par Black et Hesse qui associent toujours un support, un objectif et une temporalité. Ces derniers, s'ils n'en contestaient pas l'usage, remettent régulièrement en doute le caractère scientifique des modèles analogues et les modèles d'échelles. Black écrit même que ceux utilisés par les architectes "purement hypothétiques ne sont rien $d u$ tout, tout comme les modèles analogues imaginaires qui ne montreront jamais comment les choses fonctionnent $\gg{ }^{* *}$ en regard des modèles théoriques, qui sont les véritables piliers du raisonnement scientifique. C'est oublier que ces modèles ont construit les sciences appliquées et l'ingénierie, disciplines résolument arrimées à la méthode scientifique comme le rappelle Weisberg (Weisberg 2013).

Cette catégorisation triple constituera un instrument théorique qui, lorsqu'elle se voit replacée dans une l'histoire des modèles, permet de comprendre certains liens épistémologiques qu'entretiennent les multiples artefacts considérés comme des « modèles » au cours des âges.

\section{Une brève histoire des modèles}

Si l'expression «BIM » date des années 1970 (Eastman et al. 1974), cela ne fait véritablement qu'une quinzaine d'années que la technologie qui lui est associée aujourd'hui s'est démocratisée et son hégémonie prochaine pourrait constituer un changement de paradigme dans les pratiques de conception architecturale. Afin de comprendre la nature et l'ampleur de cette bascule, un regard rétrospectif succinct vers les outils qui l'ont précédée apparaît nécessaire.

\subsection{Usage originel des maquettes d'architecture}

Bien qu'il existe quelques exemples documentés de maquettes d'architecture antérieures au Quattrocento comme celle du temple de Delphes décrite par Hérodote ou celle, en bois, de la cathédrale de Florence à la fin du 14 siècle (Janke 1978), on associe généralement cette pratique à la figure de l'architecte italien de la Renaissance. C'est lui qui

\footnotetext{
** "Scale models and analogue models must be actually put together: a merely "hypothetical" architect's model is nothing at all, and imaginary analogue models will never show us how things work in the large." Black, M. p. 229, Notre traduction
} 
emprunte d'abord le terme de «modèle» aux Beaux-Arts durant cette période pour qualifier les réductions de bâtiments précédant l'édification du bâtiment qu'elles représentent (Rey 1998). Les maquettes étaient déjà considérées par leurs auteurs comme des « figures à reproduire ", à l'instar des modèles qui posaient pour les artistes. Alberti luimême souligne l'importance de ce type d'outil pour l'examen méticuleux du projet avant sa construction dans De Re Aedificatoria [IX.8.]. Les artefacts admettaient pourtant déjà d'autres usages comme la présentation au client, pour preuve le tableau de Domenico Cresti (1620) représentant la maquette du dôme de Saint-Pierre de Rome présentée par MichelAnge au pape Paul IV. Ces proto-modèles sont à la fois descriptifs et exploratoires, mais ne peuvent pas être qualifiés de modèles prédictifs ou d'optimisations (Dunn 2007).

On peut également qualifier de «modèles » les descriptions picturales établies par les antiquaires - spécialistes et redécouvreurs de l'antiquité au XVI et XVII siècles - dont la méthode "évolue selon une démarche comparable à celle des sciences naturelles : elle vise une même description, contrôlable et donc fiable, de ses objets " p. 63 (Choay 1996). Le terme ainsi utilisé inversait l'acception étymologique du terme de "figure à reproduire» puisque naturalistes comme antiquaires estimaient que «la copie doit être exécutée d'après nature, in situ pour les cuvres d'architecture : condition nécessaire, comme dans le cas des sciences naturelles encore, pour que l'image et sa reproduction aient une valeur. " p.64. À l'instar des modèles scientifiques dont ils s'inspirent, ces modèles analogues sont résolument descriptifs.

Les architectes, qui s'éloignent déjà de la rigueur des antiquaires par leurs inventions d'après des descriptions littéraires et leurs reconstitutions hasardeuses, participent à les rendre tout aussi exploratoires que les maquettes qu'ils produisent. Ils commencent ainsi à remettre en cause ce qui en faisait le caractère scientifique (Pérez Gómez 1987).

\subsection{L'avènement et évolution du modèle scientifique}

Le flou terminologique qui entoure le terme de modèle aujourd'hui ne peut dissimuler son acception scientifique, particulièrement fréquente. Elle est pourtant aussi récente que la physique moderne puisqu'on la doit à Niels Bohr (Rey 1998) qui l'utilise en 1913 pour qualifier sa théorie établissant une représentation du noyau atomique d'après la description de Rutherford qui sera qualifiée par la suite de modèle planétaire. Le modèle de l'atome de Bohr est un modèle analogue par excellence puisque celui-ci utilise l'équivalence des rapports entre les deux systèmes et non le simple rapport de ressemblance (Chupin 2013). Le succès de la théorie et de la représentation qui lui est associée a rapidement généralisé l'usage.

Bien que toujours descriptives, ces représentations abandonnent leur dimension exploratoire et admettent pour la première fois une dimension prédictive à laquelle seules des lois naturelles comme celles de la gravitation de Newton ou celle de l'évolution du vivant de Darwin et Wallace - rebaptisées modèles par la suite - pouvaient prétendre avant elles. Cette transition sémantique est aussi celle des supports qui ajoutent, aux modèles iconiques et analogiques, des modèles faits de mots et d'autres, de concepts mathématiques.

Le modèle scientifique actuel le plus répandu est appelé « le modèle standard ». Il s'agit d'un modèle théorique qui décrit les 17 particules élémentaires de la matière et les quatre interactions fondamentales qui les régissent. Comme celui de Bohr, premier du nom, il est essentiellement descriptif et a un grand pouvoir prédictif, raison de son succès. 


\subsection{La révolution numérique et le BIM comme horizon}

L'invention de l'ordinateur pendant la seconde guerre mondiale sera le point de départ de la cybernétique, théorie de l'information basée sur l'étude des communications dans les systèmes naturels et artificiels (Pask 1969).

Cette nouvelle mouvance imprime l'esprit logicien et ses décompositions hiérarchiques à la conception architecturale (Alexander, Ishikawa, et Silverstein 1977) qui fonctionnait encore sur les outils hérités de la période des Lumières, malgré les expériences ambitieuses du Bahaus de l'entre-deux-guerres. Ces nouveaux modèles théoriques ne suscitent pas une transformation immédiate des outils de conception et les architectes attendront les années 1990 et une programmation géométrique dénuée de formalisme mathématique (Varenne 2012), pour voir peu à peu les ordinateurs s'installer dans leurs agences. Le « Paperless Studio » de l'université Columbia, qui prône l'utilisation exclusive de l'ordinateur, est encore largement considéré comme excentrique en 1992.

Depuis, de multiples innovations technologiques ont continué à bousculer la profession tant est si bien que l'on ne remet plus en question la profondeur des mutations disciplinaires que le numérique a déjà provoquées et provoquera encore. Antoine Picon comparait déjà il y a près de dix ans l'adoption de ces nouveaux outils à la démocratisation du plan, de la coupe et de l'élévation à la Renaissance, indissociables de l'avènement des figures de l'architecte et de l'ingénieur (Picon 2010). Parmi les nombreux outils de modélisation développés depuis ce qui est désormais qualifié de " révolution numérique ", le BIM est à la fois un nouvel outil et un nouveau processus, et semble être celui d'entre eux qui convient le mieux à la comparaison.

Les maquettes BIM (2 ou 3) sont des modèles numériques sans échelle qui permettent de visualiser le bâtiment et de produire la plupart des représentations classiques de la construction (plans, coupes, élévations). La plupart des définitions de ces modèles précisent à la fois leur capacité d'analyse de données quantitatives et de partage entre différents acteurs, ce qui doit permettre leur modification itérative et in fine leur amélioration. Elles tendent donc à confondre les modèles exploratoires et descriptifs des architectes, les modèles d'optimisation des ingénieurs et les modèles prédictifs des physiciens. Cette superposition de tous les objectifs du premier volet de la classification d'Echenique explique la difficulté de toute démarche épistémologique à leur égard.

\section{Discussion}

Devant la résistance des artefacts aux catégorisations proposées, trois positions théoriques antagonistes peuvent être entreprises. Dans un premier temps, on peut remettre en cause la classification établie sur la base de son incapacité à s'appliquer à de telles modélisations. C'est un écueil récurrent de toute entreprise de catégorisation où chaque spécimen récalcitrant semble être l'occasion à la création d'une nouvelle catégorie qui, à outrance, aboutit à la création d'un taxon par cas rendant la classification stérile de fait.

La seconde consiste à considérer les modèles BIM comme la contraction de différents modèles archétypiques dont la classification sera moins hasardeuse. Une telle hypothèse permet effectivement d'analyser cet outil par sa dissection méthodique, distinguant de facto les modèles scientifiques et non scientifiques qui le composent. À l'instar des vivisections qui causent régulièrement la mort de leur objet d'étude, il est complexe de décomposer les modèles BIM, décrits comme des modèles « intégrés », uniques, sans en détruire l'essence même. En outre, si découper une souris peut aider l'observation du fonctionnement interne de l'animal, une telle opération ne nous informera pas sur son comportement social au contact de ses semblables. De même, la décomposition épistémologique des maquettes 
BIM, si elle est possible, ne nous apprendra que peu de chose sur l'usage réel que les architectes en font et sur les biais auxquels de tels outils peuvent les confronter.

La dernière attitude consiste à interroger la nature même de l'objet étudié. Est-ce véritablement un modèle tel que défini? On peut objecter qu'à l'instar des autres modélisations architecturales, un modèle BIM n'est la représentation d'aucune réalité observée. Ces derniers décrivent effectivement une réalité qui pourrait exister dans le futur mais les prédictions qu'il est susceptible de produire ne s'appliquent que dans l'éventualité où le bâtiment serait effectivement construit et non seulement relatif à la justesse de la modélisation. Cependant, à la différence des représentations spéculatives traditionnelles qui assument prudemment leur écart vis-à-vis de la réalité, le modèle BIM se considère rigoureusement identique au bâtiment futur qu'il décrit. C'est précisément le glissement de la représentation imparfaite du projet censée induire la réflexivité chez le concepteur à la simulation mesurable du bâtiment futur que l'on observe dans les écoles et les agences depuis une vingtaine d'années (Chupin et Lequay 2001; Scheer 2014).

Cette approche est d'ailleurs revendiquée par les défenseurs de l'outil qui expliquent qu' "un modèle peut être décrit par son contenu (quels objets il décrit) ou ses capacités (quels types d'information il peut supporter). Cette dernière approche est préférable, car elle définit ce que vous pouvez faire avec le modèle [...]. ${ }^{\dagger \dagger}$ " (Eastman et al. 2018). Il ne s'agit plus de représenter le projet, mais d'en simuler les caractéristiques. Cette crise de la représentation avait déjà été décrite par Jean Baudrillard comme menant au simulacre :

"Telle est la simulation, en ce qu'elle s'oppose à la représentation. Celle-ci part du principe d'équivalence du signe et du réel (même si cette équivalence est utopique, c'est un axiome fondamental). La simulation part à l'inverse de l'utopie du principe d'équivalence, part de la négation radicale du signe comme valeur, par du signe comme réversion et mise à mort de toute référence. Alors que la représentation tente d'absorber la simulation en l'interprétant comme fausse représentation, la simulation enveloppe tout l'édifice de la représentation lui-même comme simulacre. " p.16 (Baudrillard 1981)

Les maquettes BIM ne sont donc pas tant des modèles, que des simulats puisqu'en tant que simulacre, elles n'entretiennent plus guère de rapport avec la réalité tout en prétendant offrir l'intermédiaire parfait avec les situations de la réalité. Reprenant les néologismes $\mathrm{du}$ philosophe, nous pourrions alors appeler ces simulations des « hypermodèles » en ce qu'ils " génèrent un réel sans origine ni réalité : hyperréel » p.10 (Baudrillard 1981). Sans un rapport au monde, il est dès lors probablement moins utile de continuer à interroger le caractère scientifique de ces objets qui n'ont qu'un rapport feint avec le réel et qui sont, en fait, parfaitement autonomes et donc rigoureusement irréfutables.

La carte si détaillée d'un territoire qu'elle le recouvre intégralement décrite par Jorge Luis Borges et reprise par Baudrillard comme parfaite allégorie de la simulation reste féconde. " C'est désormais la carte qui précède le territoire [...] C'est elle qui engendre le territoire ", tout comme la maquette BIM précède et engendre le bâtiment. Une fois bâti, en revanche, le projet correspond de moins en moins à sa simulation et davantage à l'image du " territoire dont les lambeaux pourrissent lentement sur l'étendue de la carte. C'est le réel, et non la carte, dont des vestiges subsistent ça et là ». p. 10.

\footnotetext{
†” A building model can be described by its content (what objects it describes) or its capabilities (what kinds of information requirements it can support). The latter approach is preferable, because it defines what you can do with the model". p.13, Eastman et al., Notre traduction.
} 


\section{Bibliographie}

Baudrillard, Jean. 1981. Simulacres et simulation. Paris: Galilée.

Black, Max. 1962. Models and Metaphors: Studies in Language and Philosophy. $1981^{\mathrm{e}}$ éd. Ithaca: Cornell Univ. Press.

Choay, Françoise. 1996. L'allégorie du patrimoine. Paris: Seuil.

Chudy, Ondrej et Jan Gasparek. 2017. « What Is BIM and Why Do You Need It? » TMD STUDIO LTD.

Chupin, Jean-Pierre. 2013. Analogie et théorie en architecture: de la vie, de la ville et de la conception, même. Gollion: Infolio.

Chupin, Jean-Pierre et Hervé Lequay. 2001. « Escalade analogique et plongée numérique (Entre l'atelier tectonique et le studio virtuel dans l'enseignement du projet) ». Les Cahiers de la recherche architecturale et urbaine (7):21 à 30 .

Dunn, Nick. 2007. The Ecology of the Architectural Model. Oxford; New York: Peter Lang.

Eastman, Charles et al. 1974. « An Outline of the Building Description System. Research Report No. 50 ».

Eastman, Charles M., Paul M. Teicholz, Rafael Sacks, et Ghang Lee. 2018. « The Future: Building with BIM ». P. 364-97 in BIM Handbook: a Guide to Building Information Modeling for Owners, Managers, Designers, Engineers and Contractors. John Wiley \& Sons, Ltd.

Echenique, Marcial. 1968. Models: A Discussion. Cambridge (16 Brooklands Ave., Cambridge): University of Cambridge Dept. of Architecture.

Hesse, Mary B. 1970. Models and Analogies in Science. Notre Dame, Ind.: University of Notre Dame Press.

Janke, Rolf. 1978. Architectural Models. New York: Architectural Book Pub. Co.

Nouvel, Pascal. 2002. « Modèles \& métaphores ». in Enquête sur le concept de modèle, Science, histoire et société. Paris: Presses universitaires de France.

Pask, Gordon. 1969. « The Architectural Relevance of Cybernetics ». Architectural Design 10.

Pérez Gómez, Alberto. 1987. L'architecture et la crise de la science moderne. Trad. 1995. Liège: P. Mardaga.

Picon, Antoine. 2010. Culture Numérique et Architecture : Une Introduction. Basel:

Birkhäuser. 
Pocobelli, Danae Phaedra, Jan Boehm, Paul Bryan, James Still, et Josep Grau-Bové. 2018. « BIM for heritage science: a review ». Heritage Science 6(1):30.

Rey, Alain, éd. 1998. Dictionnaire historique de la langue française. Paris: Le Robert.

Scheer, David R. 2014. The Death of Drawing: Architecture in the Age of Simulation. London; New York: Routledge.

Varenne, Franck. 2012. « Modèles et simulations en design et architecture ».

Weisberg, Michael. 2013. Simulation and similarity: using models to understand the world. New York: Oxford University Press. 